PROCEEDINGS OF THE

AMERICAN MATHEMATICAL SOCIETY

Volume 139, Number 1, January 2011, Pages 163-176

S 0002-9939(2010)10454-9

Article electronically published on June 30, 2010

\title{
REFLEXIVITY AND HYPERREFLEXIVITY OF BOUNDED $N$-COCYCLES FROM GROUP ALGEBRAS
}

\author{
EBRAHIM SAMEI
}

(Communicated by Nigel J. Kalton)

\begin{abstract}
We introduce the concept of reflexivity for bounded $n$-linear maps and investigate the reflexivity of $\mathcal{Z}^{n}\left(L^{1}(G), X\right)$, the space of bounded $n$ cocycles from $L^{1}(G)^{(n)}$ into $X$, where $L^{1}(G)$ is the group algebra of a locally compact group $G$ and $X$ is a Banach $L^{1}(G)$-bimodule. We show that $\mathcal{Z}^{n}\left(L^{1}(G), X\right)$ is reflexive for a large class of groups including groups with polynomial growth, IN-groups, maximally almost periodic groups, and totally disconnected groups. If, in addition, $G$ is amenable and $X$ is the dual of an essential Banach $L^{1}(G)$-bimodule, then we show that $\mathcal{Z}^{1}\left(L^{1}(G), X\right)$ satisfies a stronger property, namely hyperreflexivity. This, in particular, implies that $\mathcal{Z}^{1}\left(L^{1}(G), L^{1}(G)\right)$ is hyperreflexive.
\end{abstract}

The concept of reflexivity for linear subspaces of bounded operators on Banach spaces has its origin in operator theory. Let $X$ be a Banach space, and let $A \subset B(X)$ be an algebra of bounded operators on $X$. Let Lat $A$ denote the set of the closed subspaces of $X$ invariant under $A$; i.e. for every $T \in A$ and $I \in$ Lat $A$ we have $T(I) \subset I$. We say that $A$ is reflexive if $A$ is the algebra generated by Lat $A$; i.e. every bounded operator satisfying $T(I) \subset I$ for every $I \in$ Lat $A$ belongs to $A$. This concept is closely related to the well-known invariant subspaces problem: whether a bounded operator $T \in B(X)$ has an invariant subspace.

In 10, D. R. Larson generalized the concept of reflexivity, both algebraically and topologically, to subspaces of $B(X, Y)$ for Banach spaces $X$ and $Y$. One motivation was to study the local behavior of derivations from a Banach algebra $A$ to a Banach $A$-bimodule $X$. The main question that one asks is, for which algebras every so-called "local derivation" is a derivation, or equivalently, which algebras have algebraically reflexive derivation spaces? One can also ask the topological version of this question; i.e. when is the linear space of bounded derivations reflexive [10]?

In the last two decades, the question of (algebraic) reflexivity of the derivation space has received considerable attention from various researchers, and some very interesting results have been obtained. In [9, R. D. Kadison showed that bounded local derivations from a von Neumann algebra into any of its dual bimodules are derivations. Kadison's result was generalized later by showing that the space of bounded derivations from a $\mathrm{C}^{*}$-algebra into any of its modules is both algebraically

Received by the editors January 3, 2010 and, in revised form, February 20, 2010.

2010 Mathematics Subject Classification. Primary 47B47, 43A20.

Key words and phrases. Reflexivity, hyperreflexivity, $n$-cocycles, $n$-hyperlocal maps, the derivation space, group algebras, groups with polynomial growth.

This work was partially supported by an NSERC Discovery Grant.

(C)2010 American Mathematical Society Reverts to public domain 28 years from publication 
and topologically reflexive $([8,[14)$. On the other hand, it was shown in [11] that every local derivation on $B(X)$, for a Banach space $X$, is a derivation. For further results see [3, 7, 14], 15, and reference therein.

One important example of a Banach algebra in harmonic analysis is the group algebra $L^{1}(G)$ of a locally compact group $G$. In [14, the author investigated the reflexivity of the derivation space $\mathcal{Z}^{1}\left(L^{1}(G), X\right)$ from $L^{1}(G)$ into a Banach $L^{1}(G)$ bimodule $X$ and show that it is reflexive if $G$ is a SIN or a totally disconnected group [14, Theorem 8.8]. The main idea used there was to introduce the concept of bounded approximately local derivations and show that they have to be derivations. In [3, Corollary 5.3], J. Alaminos, J. Extremera, and A. R. Villena showed that in the case where $X=L^{1}(G)$ and $G$ is an amenable SIN group, $\mathcal{Z}^{1}\left(L^{1}(G), L^{1}(G)\right)$ satisfied a stronger condition, namely hyperreflexivity. Their result depends on some nice and intriguing estimates they obtained in an earlier article 2].

The purpose of this article is to investigate further the reflexivity properties related to $L^{1}(G)$. We take two directions: the reflexivity for higher cohomology and the hyperreflexivity of $\mathcal{Z}^{1}\left(L^{1}(G), X\right)$. It is natural to consider the reflexivity for subspaces of $n$-linear maps. In the context of Hochschild cohomology, an important subspace of bounded $n$-linear maps is the space of bounded $n$-cocycles. A weaker version of higher reflexivity was studied in [16, where it was shown that, for a $\mathrm{C}^{*}$-algebra $A$ and Banach $A$-bimodule $X$, local $n$-cocycles from $A^{(n)}$ to $X$ are $n$-cocycle [16. Theorem 4.4]. We pose similar question with regard to bounded $n$-cocycles from $L^{1}(G)^{(n)}$.

In Section 2, we formulate the definition for both algebraical and topological reflexivity of subspaces of bounded $n$-linear maps. We then recall the class of Banach algebras with property $(\mathbb{B})$, which was introduced in $[1$ and shown to be useful in studying maps preserving zero products (Section 2.3). We show that we can characterize bounded $n$-cocycles for Banach algebras with property (BB) provided that the algebra has sufficiently many "local identities".

In [1, it is shown that $L^{1}(G)$ always has property $(\mathbb{B})$. In Section 3 we combine their result together with our results in Section 2 and the structure of groups with polynomial growth to show that the space of bounded $n$-cocycles from $L^{1}(G)^{(n)}$ into any Banach $L^{1}(G)$-bimodule is reflexive provided that $G$ has an open subgroup with polynomial growth. This class of groups also includes IN-groups, maximally almost periodic groups, and totally disconnected groups. Here we use the property of polynomial growth to show that $L^{1}(G)$ contains bounded approximate identities which are locally unital.

Section 4 is devoted to investigating the hyperreflexivity of $\mathcal{Z}^{1}\left(L^{1}(G), X\right)$. We first recall and/or generalize what we require from [3] and make some careful estimates. Then we again use the existence of locally unital bounded approximate identities in group algebras of groups with polynomial growth and show that $\mathcal{Z}^{1}\left(L^{1}(G), X\right)$ is hyperreflexive if $G$ is an amenable group with an open subgroup of polynomial growth and $X$ is the dual of an essential $L^{1}(G)$-bimodule. In particular, we deduce that $\mathcal{Z}^{1}\left(L^{1}(G), L^{1}(G)\right)$ is hyperreflexive.

\section{Preliminaries}

Let $X$ and $Y$ be Banach spaces. For $n \in \mathbb{N}$, let $X^{(n)}$ be the Cartesian product of $n$ copies of $X$, and let $L^{n}(X, Y)$ and $B^{n}(X, Y)$ be the spaces of $n$-linear maps and bounded $n$-linear maps from $X^{(n)}$ into $Y$, respectively. 
Let $A$ be a Banach algebra, and let $X$ be a Banach $A$-bimodule. An operator $D \in L(A, X)$ is a derivation if for all $a, b \in A, D(a b)=a D(b)+D(a) b$. For each $x \in X$, the operator $a d_{x} \in B(A, X)$ defined by $a d_{x}(a)=a x-x a$ is a bounded derivation, called an inner derivation. Let $Z^{1}(A, X)$ and $\mathcal{Z}^{1}(A, X)$ be the linear spaces of derivations and bounded derivations from $A$ into $X$, respectively. For $n \in \mathbb{N}$ and $T \in L^{n}(A, X)$, define

$$
\begin{aligned}
\delta_{X}^{n} T:\left(a_{1}, \ldots, a_{n+1}\right) & \mapsto a_{1} T\left(a_{2}, \ldots, a_{n}\right) \\
& +\sum_{j=1}^{n}(-1)^{j} T\left(a_{1}, \ldots, a_{j-1}, a_{j} a_{j+1}, \ldots, a_{n+1}\right) \\
& +(-1)^{n+1} T\left(a_{1}, \ldots, a_{n}\right) a_{n+1} .
\end{aligned}
$$

We write $\delta^{n}$ instead of $\delta_{X}^{n}$ when there is no fear of ambiguity. It is clear that $\delta^{n}$ is a linear map from $L^{n}(A, X)$ into $L^{n+1}(A, X)$; these maps are the connecting maps. The elements of $\operatorname{ker} \delta^{n}$ are the $n$-cocycles; we denote this linear space by $Z^{n}(A, X)$. If we replace $L^{n}(A, X)$ with $B^{n}(A, X)$ in the above, we will have the 'Banach' version of the connecting maps; we denote them with the same notation $\delta^{n}$. In this case, $\delta^{n}$ is a bounded linear map from $B^{n}(A, X)$ into $B^{n+1}(A, X)$; these maps are the bounded connecting maps. The elements of $\operatorname{ker} \delta^{n}$ are the bounded $n$-cocycles; we denote this linear space by $\mathcal{Z}^{n}(A, X)$ and $\operatorname{im} \delta^{n}$ by $\mathcal{N}^{n}(A, X)$. The quotient $\mathcal{Z}^{n}(A, X) / \mathcal{N}^{n-1}(A, X)$ is the $n^{\text {th }}$ Hochschild cohomology of $A$ into $X$. It is easy to check that $Z^{1}(A, X)$ and $\mathcal{Z}^{1}(A, X)$ coincide with our previous definition of these notations.

A Banach algebra $A$ is amenable if for any Banach $A$-bimodule $X$, every bounded derivation $D: A \rightarrow X^{*}$ is inner. By [4, Theorem 2.8.33 and Corollary 2.8.34], if $A$ is amenable, then $\mathcal{N}^{n-1}\left(A, X^{*}\right)=\mathcal{Z}^{n}\left(A, X^{*}\right)$ for every $n \in \mathbb{N}$; i.e. the $n^{\text {th }}$ Hochschild cohomology of $A$ into $X^{*}$ vanishes.

Let $G$ be a locally compact group with a fixed left Haar measure $\lambda$. The measure algebra $M(G)$ is the Banach space of complex-valued, regular Borel measures on $G$. The space $M(G)$ is identified with the (dual) space of all continuous linear functionals on the Banach space $C_{0}(G)$, with the duality specified by setting

$$
\langle\mu, f\rangle=\int_{G} f(t) d \mu(t) \quad\left(f \in C_{0}(G), \mu \in M(G)\right) .
$$

The convolution multiplication $*$ on $M(G)$ is defined by setting

$$
\langle\mu * \nu, f\rangle=\int_{G} \int_{G} f(s t) d \mu(s) d \nu(t) \quad\left(f \in C_{0}(G), \mu, \nu \in M(G)\right) .
$$

We write $\delta_{s}$ for the point mass at $s \in G$; the element $\delta_{e}$ is the identity of $M(G)$, and $l^{1}(G)$ is the subalgebra of $M(G)$ generated by the point masses. Then $M(G)$ is a unital Banach algebra and $L^{1}(G)$, the group algebra on $G$, is a closed ideal in $M(G)$ [4, Theorem 3.3.36]. Moreover, $M(G)=L^{1}(G)=l^{1}(G)$ if and only if $G$ is discrete. The celebrated theorem of B. E. Johnson states that $G$ is amenable if and only if $L^{1}(G)$ is amenable [4, Theorem 5.6.42].

\section{Approximately local N-COCyCles}

2.1. General construction. Let $X$ and $Y$ be Banach spaces, and let $n \in \mathbb{N}$. Let $\mathcal{S}$ be a linear subspace of $L^{n}(X, Y)$, for each $\tilde{x}=\left(x_{1}, \ldots, x_{n}\right) \in X^{(n)}$, let 
$\mathcal{S}(\tilde{x})=\{S(\tilde{x}) \mid S \in \mathcal{S}\}$, and let $[\mathcal{S}(\tilde{x})]$ be the norm-closure of $\mathcal{S}(\tilde{x})$. Put

$$
\operatorname{ref}_{a}(\mathcal{S})=\left\{T \in L^{n}(X, Y) \mid T(\tilde{x}) \in \mathcal{S}(\tilde{x}), \text { for each } \tilde{x} \in X^{(n)}\right\} ;
$$

and if $\mathcal{S} \subseteq B^{n}(X, Y)$, put

$$
\operatorname{ref}(\mathcal{S})=\left\{T \in B^{n}(X, Y) \mid T(\tilde{x}) \in[\mathcal{S}(\tilde{x})] \text {, for each } \tilde{x} \in X^{(n)}\right\} .
$$

Suppose that $\mathcal{S} \subseteq L^{n}(X, Y)$. Then $\mathcal{S}$ is algebraically reflexive if $\mathcal{S}=\operatorname{ref}_{a}(\mathcal{S})$, and when $\mathcal{S} \subseteq B^{n}(X, Y)$, it is reflexive if $\mathcal{S}=\operatorname{ref}(S)$.

In order to investigate reflexivity of $\mathcal{Z}^{n}(A, X)$ for a Banach algebra $A$ and a Banach $A$-bimodule $X$, we need to introduce the concept of "approximately local $n$-cocycles". This is basically the generalization of [14, Definition 3.1] to the higher dimensions.

Definition 2.1. Let $A$ be a Banach algebra, and let $X$ be a Banach $A$-bimodule. For $n \in \mathbb{N}$, an $n$-linear map $T$ from $A^{(n)}$ into $X$ is called an approximately local $n$-cocycle if, for each $\tilde{a}=\left(a_{1}, \ldots, a_{n}\right) \in A^{(n)}$, there is a sequence $T_{\tilde{a}, n}$ of $n$-cocycles from $A^{(n)}$ into $X$ such that $T(\tilde{a})=\lim _{n \rightarrow \infty} T_{\tilde{a}, n}(\tilde{a})$. If, in addition, $T$ is bounded, we say that $T$ is a bounded approximately local $n$-cocycle.

It is clear that each element of $\operatorname{ref}\left[\mathcal{Z}^{n}(A, X)\right]$ is a bounded approximately local $n$-cocycle, but the converse may not be true, since the n-cocycles $T_{\tilde{a}, n}$ considered above need not be bounded.

2.2. $n$-hyperlocal maps. Let $A$ be a Banach algebra, and let $X$ be a Banach $A$-bimodule. We recall from [16, Definition 2.1] that, for $n \in \mathbb{N}$, an $n$-linear map $T$ from $A^{(n)}$ into $X$ is $n$-hyperlocal if, for $a_{0}, \ldots, a_{n+1} \in A$,

$$
a_{0} a_{1}=a_{1} a_{2}=\cdots=a_{n} a_{n+1}=0 \text { implies } a_{0} T\left(a_{1}, \ldots, a_{n}\right) a_{n+1}=0 .
$$

For $n=1$, 1-hyperlocal maps are simply called hyperlocal maps or hyperlocal operators.

It is easy to see that every (approximately local) $n$-cocycle is an $n$-hyperlocal map. We would like to know when the converse holds, i.e. what condition we should put on an $n$-hyperlocal map to force it to be an $n$-cocycle. The following proposition that is proven in 15 states some sufficient conditions for a bounded $n$-hyperlocal map to be an $n$-cocycle. This is critical for us to obtain our result.

Proposition 2.2 ([16, Proposition 2.2]). Let A be a unital Banach algebra with unit 1 which satisfies the following two conditions:

(i) For every unital Banach A-bimodule $X$, a bounded operator $D: A \rightarrow X$ is a left multiplier if and only if $b a=0$ implies $D(b) a=0$.

(ii) For every unital Banach A-bimodule $X$, a bounded operator $D: A \rightarrow X$ is hyperlocal if and only if

$$
D(a c b)-a D(c b)-D(a c) b+a D(c) b=0
$$

for all $a, b, c \in A$.

Let $X$ be a unital Banach $A$-bimodule, let $n \in \mathbb{N}$, and let $T \in B^{n}(A, X)$ be an $n$-hyperlocal map such that $T\left(a_{1}, \ldots, a_{n}\right)=0$ if any one of $a_{1}, \ldots, a_{n}$ is 1 . Then $T$ is an $n$-cocycle. 
2.3. Banach algebras with the property $(\mathbb{B})$. We say that a Banach algebra $A$ has the property $(\mathbb{B})$ if, for any Banach space $X$, every continuous bilinear map $\varphi: A \times A \rightarrow X$ with the property that

$$
a, b \in A, a b=0 \text { implies } \varphi(a, b)=0
$$

is of the form of

$$
\varphi(a b, c)=\varphi(a, b c) \quad(a, b, c \in A) .
$$

Banach algebras with the property $(\mathbb{B})$ are introduced in $[$, Definition 2.2] and are shown to be very useful in studying local homomorphisms and local derivations. In [1. Section 2], various hereditary properties of Banach algebras with the property $(\mathbb{B})$ have been studied. What we require for our purpose is the connection between the property $(\mathbb{B})$ for $A$ and its unitization which we study in this section. We recall that the unitization of $\mathrm{A}$ is $A^{\sharp}:=A \oplus \mathbb{C}$ with multiplication

$$
(a, \lambda)(b, \mu)=(a b+a \mu+b \lambda, \lambda \mu) \quad(a, b \in A, \lambda, \mu \in \mathbb{C})
$$

and norm

$$
\|(a, \lambda)\|=\|a\|+|\lambda| \quad(a \in A, \lambda \in \mathbb{C}) .
$$

Thus $A^{\sharp}$ is a unital Banach algebra with unit $(0,1)$, which is denoted by 1 if there is no chance of ambiguity. Also $A$ is a closed two-sided ideal of $A^{\sharp}$ with the codimension 1.

It is shown in [1, Proposition 2.5] that if $A^{\sharp}$ has the property $(\mathbb{B})$ and $\overline{A^{2}}=A$, then so does $A$. The following theorem demonstrates the partial converse to the above statement. Our argument depends heavily on the existence of sufficiently many "local identities". This idea was successfully applied in [14.

Definition 2.3. Suppose that $A$ is a Banach algebra and $a \in A$. We say that $a$ has a left (right) identity in $A$ if for some $b \in A, b a=a(a b=a)$. We say that $A$ is approximately locally unital if there are subsets $A_{l}$ and $A_{r}$ of $A$ such that $A$ is the closed linear span of both $A_{l}$ and $A_{r}$ and each element of $A_{l}$ and $A_{r}$ has a left identity and a right identity in $A$, respectively.

Theorem 2.4. Let $A$ be an approximately locally unital Banach algebra with the property $(\mathbb{B})$. Then $A^{\sharp}$ has also the property $(\mathbb{B})$.

Proof. Let $X$ be a Banach space, and let $\varphi$ be a continuous bilinear map from $A^{\sharp} \times A^{\sharp}$ into $X$ that satisfies the following condition:

$$
a b=0 \text { implies } \varphi(a, b)=0 \quad\left(a, b \in A^{\sharp}\right) .
$$

By hypothesis,

$$
\varphi(a b, c)=\varphi(a, b c) \quad(a, b, c \in A) .
$$

Since $A$ is approximately locally unital, there are subsets $A_{l}$ and $A_{r}$ of $A$ such that $A$ is the closed linear span of both $A_{l}$ and $A_{r}$ and each element of $A_{l}$ and $A_{r}$ has a left identity and a right identity in $A$, respectively. Now take $b \in A_{l}$ and $e \in A$ with $e b=b$. Then $(e-1) b c=0$, and so, by the assumption on $\varphi$, for every $a \in A^{\sharp}$, $\varphi(a e-a, b c)=0$. Thus $\varphi(a e, b c)=\varphi(a, b c)$. This, together with (2.1), shows that

$$
\varphi(a, b c)=\varphi(a e, b c)=\varphi(a e b, c)=\varphi(a b, c) \quad\left(b \in A_{l}, c \in A\right) .
$$

Since $A$ is the closed linear span of $A_{l}$,

$$
\varphi(a, b c)=\varphi(a b, c) \quad(b, c \in A) .
$$


Now let $c \in A^{\sharp}, b \in A_{r}$ and $e \in A$ with $b e=b$. Since $b(e c-c)=0$, by the assumption on $\varphi, \varphi(a b, e c-c)=0$. Therefore, from (2.2),

$$
\begin{aligned}
\varphi(a, b c) & =\varphi(a, b e c) \\
& =\varphi(a b, e c) \\
& =\varphi(a b, e c-c)+\varphi(a b, c) \\
& =\varphi(a b, c) .
\end{aligned}
$$

Hence, for $\lambda \in \mathbb{C}$,

$$
\begin{aligned}
\varphi(a,(b+\lambda) c) & =\varphi(a, b c)+\varphi(a, \lambda c) \\
& =\varphi(a b, c)+\varphi(a \lambda, c) \\
& =\varphi(a(b+\lambda), c) .
\end{aligned}
$$

The final result follows from the fact that $A$ is the closed linear span of $A_{r}$.

We are now ready to state the main result of this section.

Theorem 2.5. Let $A$ be an approximately locally unital Banach algebra with the property $(\mathbb{B})$. Then:

(i) $A^{\sharp}$ satisfies conditions ( $i$ ) and (ii) of Proposition 2.2 ,

(ii) For any Banach $A$-bimodule $X$ and $n \in \mathbb{N}$, every bounded approximately local $n$-cocycle $T$ from $A^{(n)}$ into $X$ is an $n$-cocycle. In particular, $\mathcal{Z}^{n}(A, X)$ is reflexive and $\operatorname{ref}_{a}\left[Z^{n}(A, X)\right] \cap B^{n}(A, X)=\mathcal{Z}^{n}(A, X)$.

Proof. (i) It follows from Theorem 2.4 and [1, Theorem 4.5].

(ii) We can extend $X$ to a Banach $A^{\sharp}$-bimodule by defining $1 x=x 1=x$. Let $\sigma: L^{n}(A, X) \rightarrow L^{n}\left(A^{\sharp}, X\right)$ be a linear map defined by

$$
\sigma(T)\left(a_{1}+\lambda_{1}, \ldots, a_{n}+\lambda_{n}\right)=T\left(a_{1}, \ldots, a_{n}\right),
$$

for $a_{1}, \ldots, a_{n} \in A$ and $\lambda_{1}, \ldots, \lambda_{n} \in \mathbb{C}$. It is straightforward to check that $T \in$ $L^{n}(A, X)$ is an $n$-cocycle if and only if $\sigma(T)$ is an $n$-cocycle. Now let $T \in B^{n}(A, X)$ be a bounded approximately local $n$-cocycle, and let $\left(a_{1}+\lambda_{1}, \ldots, a_{n}+\lambda_{n}\right) \in A^{\sharp(n)}$. By the assumption on $T$, for $\tilde{a}=\left(a_{1}, \ldots, a_{n}\right) \in A^{(n)}$, there is a sequence of $n$ cocycle $\left\{T_{\tilde{a}}^{k}\right\}$ from $A^{(n)}$ into $X$ such that

$$
T\left(a_{1}, \ldots, a_{n}\right)=\lim _{k \rightarrow \infty} T_{\tilde{a}}^{k}\left(a_{1}, \ldots, a_{n}\right) .
$$

Thus

$$
\begin{aligned}
\sigma(T)\left(a_{1}+\lambda_{1}, \ldots, a_{n}+\lambda_{n}\right) & =T\left(a_{1}, \ldots, a_{n}\right) \\
& =\lim _{k \rightarrow \infty} T_{\tilde{a}}^{k}\left(a_{1}, \ldots, a_{n}\right) \\
& =\lim _{k \rightarrow \infty} \sigma\left(T_{\tilde{a}}^{k}\right)\left(a_{1}+\lambda_{1}, \ldots, a_{n}+\lambda_{n}\right) .
\end{aligned}
$$

Hence $\sigma(T)$ is a bounded approximately local $n$-cocycle, and so it is a bounded $n$-hyperlocal map. Moreover,

$$
\sigma(T)\left(a_{1}+\lambda_{1}, \ldots, a_{n}+\lambda_{n}\right)=0
$$

if any one of $a_{1}, \ldots, a_{n}$ is 0 . Thus, by part (i) and Proposition 2.2, $\sigma(T)$ is an $n$-cocycle. Therefore $T$ is an $n$-cocycle. 


\section{REFLEXIVITY OF BOUNDED $n$-COCYCLES FROM GROUP ALGEBRAS}

In this section, we investigate bounded approximately local $n$-cocycles from the group algebra $L^{1}(G)$. To this end, the class of groups with polynomial growth plays an important role.

3.1. Groups with polynomial growth. A locally compact group $G$ has polynomial growth if for every compact neighborhood $U$ of the identity element in $G$, there exist $C>0$ and $n=n(U) \in \mathbb{N}$ such that, for all $m \in \mathbb{N}$,

$$
\lambda\left(U^{m}\right) \leq C m^{n(U)}
$$

where $\lambda$ is the left Haar measure on $G$ and

$$
U^{m}=\left\{u_{1} \cdots u_{m}: u_{i} \in U, i=1, \ldots, m\right\} .
$$

It is immediate that compact groups are of polynomial growth. More generally, every locally compact group $G$ with the property that the conjugacy class of every element in $G$ is pre-compact has polynomial growth [13. Theorem 12.5.17]. Also every nilpotent group (hence an abelian group) has polynomial growth [13. Theorem 12.5.17].

An important property of groups with polynomial growth is the existence of the functional calculus of smooth functions for some elements of $L^{1}(G)$, shown in 6 ] (see also [5] and [12]), as we briefly describe below.

Let $G$ be a compactly generated locally compact group with polynomial growth. Then there exists a compact symmetric neighborhood $U$ of the identity $e$ in $G$, a constant $C>0$, and $D \in \mathbb{N}$ such that $G=\bigcup_{n=1}^{\infty} U^{n}$ and $\lambda\left(U^{n}\right) \leq C n^{D}$ for all $n \in \mathbb{N}$. We define $\tau: G \rightarrow[1, \infty)$ by

$$
\tau(x)=\inf \left\{n \in \mathbb{N}: x \in U^{n}\right\} \text { for } x \neq e, \quad \tau(e)=1
$$

Now let $\alpha, \beta$, and $\gamma$ be positive real numbers satisfying

$$
0<\alpha<\beta<1 \text { and } 0<\frac{\alpha}{\beta}<\gamma<1
$$

We define the (Frechet) algebra $L_{\beta}(G)$ to be

$$
L_{\beta}(G)=\left\{f \in L^{1}(G): \int_{G}|f(x)| e^{C \tau(x)^{\alpha}}<\infty \text { for every } C>0\right\}
$$

and $A_{\gamma}$ to be the space of all periodic $\mathrm{C}^{\infty}$-functions $\varphi \in L^{1}[0,2 \pi]$ whose Fourier transform $\widehat{\varphi}$ satisfies

$$
\sum_{n \in \mathbb{Z}}|\widehat{\varphi}(n)| e^{|n|^{\gamma}}<\infty
$$

Take $f=f^{*} \in L^{2}(G) \cap L_{\beta}(G)$, where $f^{*}(t)=\overline{f\left(t^{-1}\right)}$ and $\varphi \in A_{\gamma}$ with $\varphi(0)=0$. It is shown in [6, Section 4.6] that the sum

$$
\varphi\{f\}=\sum_{n \in \mathbb{Z}} u(\text { inf }) \widehat{\varphi}(n)
$$

converges in $L^{1}(G)$. Here $u($ inf $)=\sum_{k=1}^{\infty} \frac{(\text { inf })^{* k}}{k !}$ where we denote $h^{* k}$ for the $k$-th convolution power of $h \in L^{1}(G)$ ([6, Section 4.3]). Since $A_{\gamma}$ is an algebra under pointwise multiplication, for every $\varphi, \psi \in A_{\gamma}$ with $\varphi(0)=\psi(0)=0, \varphi \psi \in A_{\gamma}$ 
operates on every element $f=f^{*} \in L^{2}(G) \cap L_{\beta}(G)$ as well. Moreover, as it is shown in [6, Section 4.7],

$$
(\varphi \psi)\{f\}=\varphi\{f\} * \psi\{f\} .
$$

3.2. Reflexivity of bounded $n$-cocycles. The functional calculus for a compactly generated locally compact group $G$ with polynomial growth is a powerful tool in studying its group algebra. For example, using this idea, it is shown in 6, Theorem 5.6] that $L^{1}(G)$ has the Wiener property. This was achieved by constructing a certain well-behaved bounded approximate identity for $L^{1}(G)$.

In the following lemma, we expand the ideas in the proof of [6, Theorem 5.6] and use the existence of functional calculus for groups with polynomial growth to construct "locally unital" bounded approximate identities in their group algebras. We will use this fact crucially later on to obtain our main results.

Lemma 3.1. Let $G$ be a locally compact group with an open subgroup which has polynomial growth. Then there are bounded approximate identities $\left\{\varphi_{i}\right\}$ and $\left\{\psi_{i}\right\}$ in $L^{1}(G)$ such that, for every $i, \varphi_{i} * \psi_{i}=\psi_{i} * \varphi_{i}=\varphi_{i}$. In particular, $L^{1}(G)$ is approximately locally unital.

Proof. Let $H$ be an open subgroup of $G$ with polynomial growth. Let $U$ be a compact neighborhood of the identity in $H$. Then the subgroup generated by $U$ is an open, compactly generated subgroup of $G$ with polynomial growth. Hence without loss of generality, we can assume that $H$ is compactly generated.

Now let $\alpha, \beta$, and $\gamma$ be positive real numbers satisfying (3.1). Let $\left\{e_{i}\right\}_{i \in I}$ be a bounded approximate identity in $L^{1}(G)$ consisting of compactly supported continuous functions such that

$$
e_{i}^{*}=e_{i} \text { for all } i \in I \text { and } \bigcup_{i \in I} \operatorname{supp} e_{i} \subset V,
$$

where $V$ is a fixed compact, symmetric neighborhood of the identity in $G$. In particular, for every $i, e_{i} \in L^{2}(G) \cap L_{\beta}(G)$. Then, by [6, Section 4.5], there are $\varphi, \psi \in A_{\gamma}$ such that:

(i) Both $\varphi$ and $\psi$ vanish on some neighborhoods of 0 and $2 \pi$.

(ii) $0 \leq \varphi \leq 1$ and $0 \leq \psi \leq 1$.

(iii) $\psi=1$ on $\operatorname{supp} \varphi$.

(iv) $\varphi(1)=\psi(1)=1$.

In this case, it is shown in [6, Section 5.3] that both $\varphi\left\{e_{i}\right\}$ and $\psi\left\{e_{i}\right\}$ are bounded approximate identities for $L^{1}(G)$ (compare (i)-(iv) above with the equations (6)-(9) in [6, page 818]). Moreover, by (3.2) and (iii),

$$
\varphi\left\{e_{i}\right\} * \psi\left\{e_{i}\right\}=\psi\left\{e_{i}\right\} * \varphi\left\{e_{i}\right\}=(\varphi \psi)\left\{e_{i}\right\}=\varphi\left\{e_{i}\right\} .
$$

Hence we are done by putting

$$
\varphi_{i}=\varphi\left\{e_{i}\right\} \text { and } \psi_{i}=\psi\left\{e_{i}\right\} \text { for all } i \in I .
$$

Finally if we let

$$
L^{1}(G)_{l}=\left\{\varphi_{i} * f: f \in L^{1}(G), i \in I\right\}
$$

and

$$
L^{1}(G)_{r}=\left\{f * \varphi_{i}: f \in L^{1}(G), i \in I\right\},
$$

then $L^{1}(G)_{l}$ and $L^{1}(G)_{r}$ satisfy the assumption of Definition 2.3 for $A=L^{1}(G)$. Thus $L^{1}(G)$ is locally approximately unital. 
The result of Section 2 (especially Theorem 2.5) and the preceding lemma give us what we need to characterize bounded approximate local $n$-cocycles from $L^{1}(G)^{n}$ whenever $G$ has an open subgroup with polynomial growth.

Theorem 3.2. Let $G$ be a locally compact group with an open subgroup which has polynomial growth. Then, for any Banach $L^{1}(G)$-bimodule $X$ and $n \in \mathbb{N}$, every bounded approximately local n-cocycle from $L^{1}(G)^{(n)}$ into $X$ is an n-cocycle. In particular, $\mathcal{Z}^{n}\left(L^{1}(G), X\right)$ is reflexive and

$$
r e f_{a}\left[Z^{n}\left(L^{1}(G), X\right)\right] \cap B^{n}\left(L^{1}(G), X\right)=\mathcal{Z}^{n}\left(L^{1}(G), X\right) .
$$

Proof. It follows from Lemma 3.1 that $L^{1}(G)$ is approximately locally unital. Also, by $\left[1\right.$, Examples 1.3 and Theorem 2.11], $L^{1}(G)$ satisfies the property $(\mathbb{B})$. Therefore the result follows from Theorem 2.5.

We finish this section with the following corollary, which provides various classes of groups satisfying the assumption of Theorem 3.2

We recall that a locally compact group $G$ is an $I N$-group if it has a compact neighborhood of identity which is invariant under all inner automorphisms of $G$. Also $G$ is maximally almost periodic if finite-dimensional continuous unitary irreducible representations of $G$ separate its point. Finally $G$ is totally disconnected if the connected component of $G$ is the identity $e$ in $G$; i.e. the largest connected subset of $G$ containing $\{e\}$ is $\{e\}$ itself.

Corollary 3.3. Let $G$ be a locally compact group, let $X$ be a Banach $L^{1}(G)$ bimodule, and let $n \in \mathbb{N}$. Then $\mathcal{Z}^{n}\left(L^{1}(G), X\right)$ is reflexive in either of the following cases:

(i) $G$ is a group of polynomial growth;

(ii) $G$ is an IN-group;

(iii) $G$ is maximally almost periodic;

(iv) $G$ is totally disconnected.

Proof. By Theorem [3.2, it suffices to show that each class of groups satisfying any of $(i)-(i v)$ above has an open subgroup of polynomial growth. If $G$ is totally disconnected, then it is well-known that $G$ has a basis of the identity consisting of open compact subgroups. Also if $G$ is an IN-group, then it has an open subgroup with pre-compact conjugacy class [13, Theorem 12.1.31] which is of polynomial growth by [13, Theorem 12.5.17]. Finally if $G$ is maximally almost periodic, then it has a (compactly generated) open IN-subgroup [13, Theorem 12.4.16(d)]. Hence it has an open subgroup with polynomial growth since the same holds for IN-groups.

\section{HyperReflexivity of the DERIVAtion SPACE FROM GROUP ALGEBRAS}

Let $X$ and $Y$ be Banach spaces, and let $\mathcal{S}$ be a linear subspace of $B(X, Y)$. For every $T \in B(X, Y)$, we define

$$
\operatorname{dist}(T, \mathcal{S})=\inf _{S \in \mathcal{S}}\|T-S\|
$$

and

$$
\operatorname{dist}_{r}(T, \mathcal{S})=\sup _{\|x\| \leq 1} \inf _{S \in \mathcal{S}}\|T(x)-S(x)\|
$$


It is clear that $\operatorname{dist}_{r}(T, \mathcal{S}) \leq \operatorname{dist}(T, \mathcal{S})$. We say that $\mathcal{S}$ is hyperreflexive if there is $C>0$ such that

$$
\operatorname{dist}(T, \mathcal{S}) \leq C \operatorname{dist}_{r}(T, \mathcal{S})
$$

for all $T \in B(X, Y)$. When the subspace $\mathcal{S}$ is a closed reflexive subspace of $B(X, Y)$, the hyperreflexivity of $\mathcal{S}$ has an equivalent reformulation in terms of norms. If we consider the quotient space $B(X, Y) / \mathcal{S}$, then both $\operatorname{dist}(T, \mathcal{S})$ and $\operatorname{dist}_{r}(T, \mathcal{S})$ define norms on $B(X, Y) / \mathcal{S}$, the former being the natural quotient norm. Therefore $\mathcal{S}$ is hyperreflexive if and only if the norms $\operatorname{dist}(T, \mathcal{S})$ and $\operatorname{dist}_{r}(T, \mathcal{S})$ are equivalent. By the inverse-mapping theorem, this happens if and only if $\operatorname{dist}_{r}(T, \mathcal{S})$ is a complete norm.

In this section, we would like to investigate when $\mathcal{Z}^{1}\left(L^{1}(G), X\right)$ is hyperreflexive for a Banach $L^{1}(G)$-bimodule $X$.

Let $A$ be a Banach algebra, and let $X$ be a Banach $A$-bimodule. For every bounded operator operator $T: A \rightarrow X$, we can associate the following number introduced in [3, Section 4]:

$$
\operatorname{zder}(T)=\sup \{\|a T(b) c\|: a, b, c \in A, a b=b c=0,\|a\|=\|b\|=\|c\|=1\} .
$$

The following lemma is proven in [3, Lemma 5.1] for the case where $X=A$. Our proof is the generalization of their proof and is presented here for the sake of completeness.

Lemma 4.1. Let $A$ be a Banach algebra, let $X$ be a Banach $A$-bimodule, and let $T \in B(A, X)$. Then

$$
\operatorname{zder}(T) \leq \operatorname{dist}_{r}\left(T, \mathcal{Z}^{1}(A, X)\right)
$$

Proof. Let $a, b, c \in A$ with $\|a\|=\|b\|=\|c\|=1$ such that $a b=b c=0$. For every $D \in \mathcal{Z}^{1}(A, X)$,

$$
a D(b) c=D(a b) c-D(a) b c=0 .
$$

Thus

$$
\|a T(b) c\|=\|a[T(b)-D(b)] c\| \leq\|T(b)-D(b)\|,
$$

which implies that

$$
\|a T(b) c\| \leq \operatorname{dist}_{r}\left(T, \mathcal{Z}^{1}(A, X)\right) .
$$

The final result follows since

$$
\operatorname{zder}(T)=\sup \{\|a T(b) c\|: a, b, c \in A, a b=b c=0,\|a\|=\|b\|=\|c\|=1\} .
$$

We recall that, for a Banach algebra $A$, a Banach left [right] $A$-module $X$ is essential if it is the closure of $A X=\operatorname{span}\{a x \mid a \in A, x \in X\}[X A=\operatorname{span}\{x a \mid$ $a \in A, x \in X\}$ ] and a Banach $A$-bimodule $X$ is essential if it is essential both as a Banach left and right $A$-module.

Theorem 4.2. Let $G$ be a locally compact group with an open subgroup of polynomial growth. Let $X$ be an essential or the dual of an essential Banach $L^{1}(G)$ bimodule, let $T: L^{1}(G) \rightarrow X$ be a bounded operator, and let $K>0$ be such that

$$
\operatorname{zder}(T)<K \text { and }\|T\| \leq K \text {. }
$$

Then there is $M>0$ depending only on $L^{1}(G)$ and a continuous function $\zeta$ : $[0,1) \rightarrow[0, \infty)$ (independent of $T, K$, and $M)$ with $\zeta(0)=0$ such that if

$$
\alpha=\left(\frac{17^{2}}{3}+1\right)^{2}(K \operatorname{zder}(T))^{1 / 2}(2+\zeta(\operatorname{zder}(T) / K))+K \zeta(\operatorname{zder}(T) / K)<2 K,
$$


then for all $f, g \in L^{1}(G)$,

$$
\begin{aligned}
& \|T(f * g)-f * T(g)-T(f) * g\| \\
& \quad \leq\left[\left(\frac{17^{2}}{3}+1\right)^{2}(2 \alpha K)^{1 / 2}(2+\zeta(\alpha / 2 K))+2 K \zeta(\alpha / 2 K)\right. \\
& \left.\quad+\operatorname{dist}_{r}\left(T, \mathcal{Z}^{1}(A, X)\right)\right] M\|f\|\|g\| .
\end{aligned}
$$

Proof. Let $f, g, h, k, l \in L^{1}(G)_{1}=\left\{p \in L^{1}(G):\|p\| \leq 1\right\}$, and put

$$
L(g, h, k)=T(g * h * k)-T(g * h) k-g T(h * k)+g T(h) k .
$$

A similar argument to the proof of [3, Theorem 4.1] shows that there is a continuous function $\zeta:[0,1) \rightarrow[0, \infty)$, independent of $T$ and $K$, with $\zeta(0)=0$ such that if

$$
\alpha=\left(\frac{17^{2}}{3}+1\right)^{2}(K \operatorname{zder}(T))^{1 / 2}(2+\zeta(\operatorname{zder}(T) / K))+K \zeta(\operatorname{zder}(T) / K)<2 K,
$$

then

$$
\|f L(g, h, k) l\| \leq\left(\frac{17^{2}}{3}+1\right)^{2}(2 \alpha K)^{1 / 2}(2+\zeta(\alpha / 2 K))+2 K \zeta(\alpha / 2 K) .
$$

In fact, in [3, Theorem 4.1], the relation (4.2) is only proven for the case where $X=L^{1}(G)$. However a careful examination of their argument shows that it also works when $X$ is an essential or the dual of an essential Banach $L^{1}(G)$-bimodule. Suppose that $\left\{e_{i}\right\}_{i \in I}$ is any approximate identity for $L^{1}(G)$ bounded by 1 . Then (4.2) implies that for every $i \in I$,

$$
\left\|e_{i} L(g, h, k) e_{i}\right\| \leq\left(\frac{17^{2}}{3}+1\right)^{2}(2 \alpha K)^{1 / 2}(2+\zeta(\alpha / 2 K))+2 K \zeta(\alpha / 2 K) .
$$

We claim that

$$
\|L(g, h, k)\| \leq\left(\frac{17^{2}}{3}+1\right)^{2}(2 \alpha K)^{1 / 2}(2+\zeta(\alpha / 2 K))+2 K \zeta(\alpha / 2 K) .
$$

If $X$ is essential, then by Cohen's factorization theorem [4, Corollary 2.9.26], there are $p, q \in L^{1}(G)$ and $x \in X$ such that $L(g, h, k)=p x q$. So

$$
\lim _{i \rightarrow \infty} e_{i} L(g * h * k) e_{i}=\lim _{i \rightarrow \infty}\left(e_{i} * p\right) x\left(e_{i} * q\right)=p x q=L(g * h * k) .
$$

Thus (4.4) follows by letting $i \rightarrow \infty$ in (4.3). On the other hand, if $X=Y^{*}$, where $Y$ is an essential Banach $L^{1}(G)$-bimodule, then

$$
\begin{aligned}
\left\|e_{i} L(g, h, k) e_{i}\right\| & =\sup \left\{\left|\left\langle e_{i} L(g * h * k) e_{i}, y\right\rangle\right|: y \in Y,\|y\|=1\right\} \\
& =\sup \left\{\left|\left\langle L(g * h * k), e_{i} y e_{i}\right\rangle\right|: y \in Y,\|y\|=1\right\} .
\end{aligned}
$$

But similar to the preceding case, $\lim _{i \rightarrow \infty} e_{i} y e_{i}=y$ for all $y \in Y$. Thus if $\|y\|=1$, we have

$$
\begin{aligned}
|\langle L(g, h, k), y\rangle| & =\lim _{i \rightarrow \infty}\left|\left\langle L(g * h * k), e_{i} y e_{i}\right\rangle\right| \\
& =\lim _{i \rightarrow \infty}\left|\left\langle e_{i} L(g * h * k) e_{i}, y\right\rangle\right| .
\end{aligned}
$$

Hence again (4.4) follows by the preceding equality and (4.3). Therefore in any case, (4.4) holds. Now let $\left\{\varphi_{i}\right\}$ and $\left\{\psi_{i}\right\}$ be the approximate identities bounded by $N>0$ constructed in Lemma 3.1. Then, for every $D \in \mathcal{Z}^{1}\left(L^{1}(G), X\right)$,

$$
\begin{aligned}
\left\|\left(g * \varphi_{i}\right) T\left(\psi_{i}\right)\left(\varphi_{i} * k\right)\right\| & =\left\|\left(g * \varphi_{i}\right)\left[T\left(\psi_{i}\right)-D\left(\psi_{i}\right)\right]\left(\varphi_{i} * k\right)\right\| \\
& \leq N^{2}\left\|T\left(\psi_{i}\right)-D\left(\psi_{i}\right)\right\|,
\end{aligned}
$$


and so,

$$
\begin{aligned}
\left\|\left(g * \varphi_{i}\right) T\left(\psi_{i}\right)\left(\varphi_{i} * k\right)\right\| & \leq N^{2} \inf \left\{\left\|T\left(\psi_{i}\right)-D\left(\psi_{i}\right)\right\|: D \in \mathcal{Z}^{1}\left(L^{1}(G), X\right)\right\} \\
& \leq N^{3} \operatorname{dist}_{r}\left(T, \mathcal{Z}^{1}\left(L^{1}(G), X\right)\right) .
\end{aligned}
$$

Since $\varphi_{i} * \psi_{i}=\psi_{i} * \varphi_{i}=\varphi_{i}$, it follows from (4.1), (4.4), and the preceding inequality that

$$
\begin{aligned}
& \left\|T\left(g * \varphi_{i} * \varphi_{i} * k\right)-T\left(g * \varphi_{i}\right)\left(\varphi_{i} * k\right)-\left(g * \varphi_{i}\right) T\left(\varphi_{i} * k\right)\right\| \\
& \leq\left\|L\left(g * \varphi_{i}, \psi_{i}, \varphi_{i} * k\right)\right\|+\left\|\left(g * \varphi_{i}\right) T\left(\psi_{i}\right)\left(\varphi_{i} * k\right)\right\| \\
& \leq N^{3}\left[\left(\frac{17^{2}}{3}+1\right)^{2}(2 \alpha K)^{1 / 2}(2+\zeta(\alpha / 2 K))+2 K \zeta(\alpha / 2 K)+\operatorname{dist}_{r}\left(T, \mathcal{Z}^{1}\left(L^{1}(G), X\right)\right)\right] .
\end{aligned}
$$

The final result follows from letting $i \rightarrow \infty$.

We are now ready to prove the main result of this section, which states that under the assumption of Theorem 3.2, we can obtain hyperreflexivity for $\mathcal{Z}^{1}\left(L^{1}(G), X\right)$ if we further assume that $G$ is amenable and $X$ is the dual of an essential Banach $L^{1}(G)$-bimodule.

Theorem 4.3. Let $G$ be an amenable locally compact group with an open subgroup with polynomial growth. Let $X$ be the dual of an essential Banach $L^{1}(G)$-bimodule. Then $\mathcal{Z}^{1}\left(L^{1}(G), X\right)$ is hyperreflexive. In particular, $\mathcal{Z}^{1}\left(L^{1}(G), L^{1}(G)\right)$ is hyperreflexive.

Proof. First note that, by Theorem $3.2 \mathcal{Z}^{1}\left(L^{1}(G), X\right)$ is reflexive. Hence, from the remark made before Lemma $4.1 \mathcal{Z}^{1}\left(L^{1}(G), X\right)$ is hyperreflexive if we could show that the identity map on $B\left(L^{1}(G), X\right) / \mathcal{Z}^{1}\left(L^{1}(G), X\right)$ is continuous with respect to norms $\operatorname{dist}_{r}\left(\cdot, \mathcal{Z}^{1}\left(L^{1}(G), X\right)\right)-\operatorname{dist}\left(\cdot, \mathcal{Z}^{1}\left(L^{1}(G), X\right)\right)$. Suppose otherwise; i.e. there is a sequence $\left\{T_{n}\right\} \subset B\left(L^{1}(G), X\right)$ such that

$$
\operatorname{dist}_{r}\left(T_{n}, \mathcal{Z}^{1}\left(L^{1}(G), X\right)\right) \rightarrow 0 \text { as } n \rightarrow \infty
$$

but

$$
\operatorname{dist}\left(T_{n}, \mathcal{Z}^{1}\left(L^{1}(G), X\right)\right)=1 / 2 \text { for all } n \in \mathbb{N} .
$$

By replacing $T_{n}$ with $T_{n}+D_{n}$ for some suitable $D_{n} \in \mathcal{Z}^{1}\left(L^{1}(G), X\right)$, we can assume that

$$
\left\|T_{n}\right\|<1 .
$$

Suppose that $\delta_{X}^{1}: B^{1}\left(L^{1}(G), X\right) \rightarrow B^{2}\left(L^{1}(G), X\right)$ is the bounded connecting map introduced in Section 1. It follows from Lemma 4.1 and (4.5) that

$$
\operatorname{zder}\left(T_{n}\right) \rightarrow 0 \text { as } n \rightarrow \infty .
$$

Thus, by Theorem 4.2 (for $K=1$ ), there is $M>0$ and a continuous function $\zeta:[0,1) \rightarrow[0, \infty)$ with $\zeta(0)=0$ such that for $n$ large enough,

$$
\alpha_{n}=\left(\frac{17^{2}}{3}+1\right)^{2}\left(\operatorname{zder}\left(T_{n}\right)\right)^{1 / 2}\left(2+\zeta\left(\operatorname{zder}\left(T_{n}\right)\right)\right)+\zeta\left(\operatorname{zder}\left(T_{n}\right)\right)<2
$$

and

$$
\begin{aligned}
& \left\|\delta_{X}^{1} T_{n}\right\| \\
& \quad \leq M\left[\left(\frac{17^{2}}{3}+1\right)^{2}\left(2 \alpha_{n}\right)^{1 / 2}\left(2+\zeta\left(\alpha_{n} / 2\right)\right)+2 \zeta\left(\alpha_{n} / 2\right)+\operatorname{dist}_{r}\left(T_{n}, \mathcal{Z}^{1}(A, X)\right)\right] .
\end{aligned}
$$


Note that since $\zeta$ is continuous and $\operatorname{zder}\left(T_{n}\right) \rightarrow 0$ as $n \rightarrow \infty$,

$$
\lim _{n \rightarrow \infty} \alpha_{n}=0 \text {. }
$$

In particular, for $n$ large enough, we can assume that

$$
\alpha_{n}<2 \text {. }
$$

Since $L^{1}(G)$ is amenable, $\operatorname{im} \delta_{X}^{1}=\mathcal{Z}^{2}\left(L^{1}(G), X\right)$ 4, Corollary 2.8.34]. In particular, $\operatorname{im} \delta_{X}^{1}$ is closed, and so, by the open-mapping theorem, there is $L>0$ such that for every $S \in B\left(L^{1}(G), X\right)$,

$$
\operatorname{dist}\left(S, \mathcal{Z}^{1}\left(L^{1}(G), X\right)\right) \leq L\left\|\delta_{X}^{1} S\right\| .
$$

On the other hand, the continuity of $\zeta$, (4.5), (4.7), and (4.8) imply that $\left\|\delta_{X}^{1} T_{n}\right\| \rightarrow$ 0 as $n \rightarrow \infty$. Combining this with (4.9), we obtain

$$
\operatorname{dist}\left(T_{n}, \mathcal{Z}^{1}\left(L^{1}(G), X\right)\right) \rightarrow 0 \text { as } n \rightarrow \infty,
$$

which is a contradiction due to (4.6). Hence $\mathcal{Z}^{1}\left(L^{1}(G), X\right)$ is hyperreflexive. Finally we note that since $\mathcal{Z}^{1}\left(L^{1}(G), L^{1}(G)\right)=\mathcal{Z}^{1}\left(L^{1}(G), M(G)\right)$, it follows that $\mathcal{Z}^{1}\left(L^{1}(G), L^{1}(G)\right)$ is hyperreflexive.

We finish this section with the following corollary, which provides various classes of groups satisfying the assumption of Theorem 4.3 . The case where $G$ is an amenable SIN group and $X=L^{1}(G)$ has already been proven in [3, Corollary 5.3].

Corollary 4.4. Let $G$ be a locally compact group, and let $X$ be the dual of an essential Banach $L^{1}(G)$-bimodule. Then $\mathcal{Z}^{1}\left(L^{1}(G), X\right)$ is hyperreflexive in any of the following cases:

(i) $G$ is a group of polynomial growth;

(ii) $G$ is an amenable IN-group;

(iii) $G$ is an amenable maximally almost periodic group;

(iv) $G$ is an amenable totally disconnected group.

Proof. By the proof of Corollary 3.3 any of the groups in $(i)-(i v)$ has open subgroup with polynomial growth. Thus the result follows from Theorem 4.3. We note that groups with polynomial growth are amenable [13, Theorem 12.5.14].

\section{ACKNOWLEDGMENTS}

The author would like to thank Armando R. Villena for providing the preprints of the manuscripts [1, 2], and [3]. The author also would like to thank the referee for carefully reading this article and providing helpful comments.

\section{REFERENCES}

[1] J. Alaminos, M. Brear, J. Extremera, A. R. Villena, Maps preserving zero products, Studia Math. 193 (2009), no. 2, 131-159. MR2515516 (2010b:46101)

[2] J. Alaminos, J. Extremera, A. R. Villena, Approximately zero product preserving maps, Israel J Math, to appear.

[3] J. Alaminos, J. Extremera, A. R. Villena, Hyperreflexivity of the derivation space of some group algebras, Math. Z, to appear.

[4] H. G. Dales, Banach algebras and automatic continuity, New York, Oxford University Press, 2000. MR1816726 (2002e:46001)

[5] J. Dixmier, Opérateurs de rang fini dans les représentations unitaires, Publ. Math. IHES 6, 13-25 (1960) MR0136684(25:149) 
[6] G. Fendler, K. Gröchenig, M. Leinert, J. Ludwig, C. Molitor-Braun, Weighted group algebras on groups of polynomial growth, Math. Z. 245 (2003), no. 4, 791-821. MR2020712 (2004k:43008)

[7] D. Hadwin and J. Li, Local derivations and local automorphisms on some algebras, J. Operator Theory 60 (2008), no. 1, 29-44. MR2415555(2009e:47056)

[8] B. E. Johnson, Local derivations on $C^{*}$-algebras are derivations, Trans. Amer. Math. Soc, 353 (2000), 313-325. MR1783788 (2002c:46132)

[9] R. V. Kadison, Local derivations, J. Algebra 130 (1990), 494-509. MR1051316 (91f:46092)

[10] D. R. Larson, Reflexivity, algebraic reflexivity and linear interpolation, Amer. J. Math. 110 (1988), 283-299. MR935008(89d:47096)

[11] D. R. Larson and A. Sourour, Local derivations and local automorphisims of $B(X)$, Proc. Sympos. Pure Math. 51, Amer. Math. Soc., 1990, 187-194. MR.1077437 (91k:47106)

[12] J. Ludwig, Polynomial growth and ideals in group algebras, Manuscripta Math. 30 (1980), no. 3, 215-221. MR.557105 (81e:43012)

[13] T. W. Palmer, Banach algebras and general theorem of *-algebras, II. Cambridge University Press, 2001. MR 1819503 (2002e:46002)

[14] E. Samei, Approximately local derivations, J. London Math. Soc. (2) 71 (2005), no. 3, 759778. MR2132382 (2006a:46061)

[15] E. Samei, Hyper-Tauberian algebras and weak amenability of Figà-Talamanca-Herz algebras, J. Funct. Anal. 231 (2006), no. 1, 195-220. MR.2190169 (2007b:46079)

[16] E. Samei, Local properties of the Hochschild cohomology of $\mathrm{C}^{*}$-algebras, J. Australian Math. Soc. 84 (2008), 117-130. MR2469272(2010a:46130)

Department of Mathematics and Statistics, University of Saskatchewan, 106 Wiggins Road, Saskatoon, SK, Canada

E-mail address: samei@math.usask.ca 